\title{
PROGRAM
}

\section{Fourth Annual Huntington Disease Clinical Research Symposium}

\section{Organized by the Huntington Study Group}

To be held on Saturday, 16 October, 2010, in the Pavilion Ballroom at the Hyatt Regency La Jolla at Aventine, San Diego, California, USA.

This activity has been planned and implemented in accordance with the Essential Areas and Policies of the Accreditation Council for Continuing Medical Education to provide continuing medical education for physicians. The University of Rochester School of Medicine and Dentistry designates this educational activity for a maximum of 2.75 AMA PRA Category 1 Credit(s) ${ }^{\mathrm{TM}}$. Physicians should only claim credit commensurate with the extent of their participation in the activity.

The Symposium features three keynote speakers and four platform presentations by the following individuals with a panel discussion closing the session. In addition to the open panel, there will be allotted time for questions and answers after each keynote presenter.

8:00-9:00 AM

Poster viewing.

9:00-9:10 AM

INTRODUCTION_Introduction and acknowledgements.

Andrew Feigin, MD, Chair, HSG Symposia Committee and Vicki Wheelock, MD, Co-chair, HSG Symposia Committee.

9:10-9:40 AM

KEYNOTE ADDRESS-Huntington's Disease-from a Generational Curse to Hope in a Petri Dish.

Frances Saldaña. University of California, Irvine, Irvine, CA, USA.

Frances Saldaña's fight as an advocate for Huntington's Disease patients started thirty years ago, when her husband was given the fatal diagnosis of Huntington's Disease. With very vague information and no real knowledge of what Huntington's Disease was, her primary concern at the time was simply to survive and to care for her husband and her three young children. Behind her busy life was the constant reminder that her children were now "at risk" for inheriting the fatal disease. That thought inspired the urgency to advocate for HD families, for the cure, and for a treatment for Huntington's Disease. As fate would have it, all three of Frances' children inherited the mutant protein. Her youngest daughter, Marie, lost the battle to HD in November, 2009.

The movement to eradicate HD through stem cell research and clinical trials represents the catalyst of HOPE, and the knowledge that future generations will not have to fear the fatal diagnosis of what many refer to as "a generational curse".

Frances is HDSA Orange County Chapter Advocacy Chairman. She also represents the Huntington's Disease community as a member of the Sue and Bill Gross Stem Cell Research Center Patient Advocacy Committee (PAC) at UC Irvine. The Patient Advocacy Committee has been charged with providing the necessary link between the Stem Cell Research Center and the driving force for stem cell research. The committee advocates to develop the science that will lead to effective treatments for the greater public impacted by disease or disability, and bringing the promises of stem cell research to effective treatments.

The collaboration between clinical investigators, doctors, pharmaceutical companies, and Huntington's Disease families in support of stem cell research and clinical trials has been a model template for all humanity and the only hope for a cure.

9:40-10:10 AM

KEYNOTE ADDRESS-Developing a Therapy for Huntington's Disease by Reducing Expression of the Huntingtin Gene.

Don Cleveland, PhD. University of California, San Diego, La Jolla, CA, USA.

All instances of Huntington's disease are caused by triplet nucleotide CAG expansion in huntingtin. A toxic property of the mutant huntingtin is responsible for widespread brain degeneration, especially within the striatum. Thus, diminishing the synthesis of mutant huntingtin will directly target the actual cause of HD, as long as it is effective within the cells that contribute meaningfully to toxicity. To achieve this, a gene therapy is being developed, exploiting gene silencing throughout the nervous system. The approach is infusion of antisense oligonucleotides (ASOs) to catalyze destruction of the mRNAs encoding huntingtin. ASOs do not cross the blood brain barrier; hence, infusion of them into the CNS or periphery is required for selectively diminishing synthesis within the nervous system. Using either of two mouse models of HD (the BACHD and R6/2 mice), we have determined that ASO suppression of mutant huntingtin delays loss of brain mass and reverses disease symptoms. A concern for this approach is that although only one of the two Huntington's genes is mutated in Huntington's patients, the activity of both the mutant and normal gene is diminished simultaneously and loss of normal huntingtin may be deleterious. Uniform depletion of endogenous mouse huntingtin has been analyzed in mice that express only a single copy of mouse huntingtin (huntingtin hemizygous). Counterintuitively, reduction of endogenous huntingtin improves motor performance compared to wild-type littermates. 
Additionally, to best mimic human treatment conditions and determine whether loss of normal huntingtin exacerbates disease, ASOs that indistinguishably target both mouse and human huntingtin genes or that selectively target only the mouse allele have been identified. Treatment of both mouse models is underway to determine if depletion of endogenous huntingtin exacerbates or ameliorates disease. These experiments will determine if ASO gene silencing of both mutant and normal alleles is a feasible treatment for Huntington's disease.

\section{0:10-10:20 AM}

Platform Presentation-The Effect of Video GameBased Exercise on Dynamic Balance, Mobility and UHDRS Neuropsychiatric Test Scores in Individuals with Huntington's Disease.

A. Kloos, ${ }^{1}$ N. Fritz, ${ }^{1}$ S. Kostyk, ${ }^{2}$ and D. Kegelmeyer. ${ }^{1}{ }^{1}$ The Ohio State University, Department of Physical Therapy, Columbus, OH, USA and ${ }^{2}$ The Ohio State University, Department of Neurology, Columbus, OH, USA.

Background: Huntington's disease (HD) produces balance and gait impairments that increase fall risk and decrease quality of life for affected individuals.

Aims: This study examined the efficacy of an exercise program using the video game Dance Dance Revolution (DDR) to improve dynamic balance and mobility in individuals with HD.

Methods: Participants were 20 adults with HD who could ambulate $10 \mathrm{ft}$ without assistance. A cross-over control design was utilized. Subjects were assigned to experimental $(n=12)$ and control $(n=8)$ groups. After 6 weeks of usual care, the experimental group performed DDR for $45 \mathrm{~min} 2 \times /$ week for 6 weeks in their homes with a researcher. The control group performed a handheld video game. Subjects were pre-tested when entering the study and immediately prior to the intervention, and post-tested after the DDR intervention and control.

Results: Preliminary data analysis suggests a trend for improved Tinetti Mobility Test balance scores in subjects with mid-stage disease (UHDRS $=>42$ ) while early stage subjects (UHDRS $=<42$ ) showed a greater improvement on UHDRS Cognitive Assessments following the DDR intervention. Subjects showed improvements in the four-square step test after the intervention suggesting improved dynamic balance. Data analysis of ABC scale, WHOQoL, and backwards walking is ongoing. Subjects reported that the DDR game was fun, challenging, and highly motivating.

Conclusions: The preliminary results indicate that DDR is well tolerated, and may improve dynamic balance and cognition in this population. Analysis is on-going to determine if the DDR intervention improves spatiotemporal gait measures.

10:20-10:30 AM

Platform PResentation-A Randomized, Double Blind, Placebo-Controlled Pilot Study of Memantine in Huntington's Disease.

L. Medina, ${ }^{1}$ E. Pirogovsky, ${ }^{1}$ J. Goldstein, ${ }^{2}$ S. Roesch, ${ }^{1}$ A. Rosenblatt, ${ }^{3}$ R. Dubinsky, ${ }^{4}$ P. Gilbert, ${ }^{1}$ and J. Corey-Bloom. ${ }^{2}$ ${ }^{I}$ San Diego State University/University of California, San Diego Joint Doctoral Program in Clinical Psychology, San Diego, CA, USA, ${ }^{2}$ University of California, San Diego, Department of Neurology, San Diego, CA, USA, ${ }^{3}$ Johns Hopkins School of Medicine, Department of Psychiatry, Baltimore, MD, USA, and ${ }^{4}$ University of Kansas, Department of Neurology, Kansas City, KS, USA.
Background: Huntington's disease (HD), a neurodegenerative disorder resulting from the trinucleotide CAG expansion of the HD gene, is typified by cognitive, psychiatric, and motor dysfunction. Memantine is an NMDA receptor antagonist that has been shown to be effective in the treatment of Alzheimer's disease. Although not currently indicated for the treatment of $\mathrm{HD}$, memantine may have implications for the management of HD cognitive symptoms.

Methods: Fifty mild to moderate HD patients (31 women, 19 men; mean age $=47.25$, mean education $=14.54$, mean $C A G$ repeats $=45.29$, mean $\mathrm{DRS}=119.18$ ) were randomly assigned at three academic medical centers in the US to either memantine $10 \mathrm{mg}$ or identical-appearing placebo BID for 12 weeks. Both groups then received memantine for another 12 weeks. Cognitive, behavioral, functional, and motor data were collected at baseline, 3 , and 6 months.

Results: Hierarchical linear modeling demonstrated that memantine use was related to a significant $(p<0.05)$ improvement in performance on attention (Brief Test of Attention), working memory (Digit Ordering Test), and delayed recall (Hopkins Verbal Learning Test). However, memantine users did demonstrate significant $(p<0.01)$ worsening in motor symptoms on the Quantitative Neurological Examination. There were no statistically significant differences between groups on the Neuropsychiatric Inventory or Alzheimer's Disease Cooperative Study-Activities of Daily Living Scale.

Conclusions: Results indicate potential benefits of memantine on cognitive performance in $\mathrm{HD}$, but possible negative effects on motor symptoms. Larger studies will be needed to confirm and extend these findings.

10:30-10:50 AM

Break and poster viewing.

10:50-11:20 AM

KEYNOTE ADDRESS-Strategies to Treat HD Through Modification of the Huntingtin Protein.

Leslie Michels Thompson, PhD. University of California, Irvine, Irvine, CA, USA.

The synthesis of a mutated form of the Huntingtin protein is the primary cause of HD. However, the toxicity conferred by the presence of this mutant protein in the brain can be modulated by addition of other molecules and small proteins. These posttranslational modifications regulate the activity of proteins and may alter their biological function; therefore we are investigating whether modification of mutant Huntingtin (Htt) protein can influence its pathogenicity. We find that a small region of $\mathrm{Htt}$, the first 17 amino acids, can have a significant impact on $\mathrm{Htt}$ aggregation, neurotoxicity, and clearance and is likely to influence mutant $\mathrm{Htt}$ accumulation and dysregulation of nuclear activities. The Htt protein can be SUMO modified and SUMO modification itself appears relevant to HD pathogenesis in mouse tissue and human HD brain. Further, Htt can be phosphorylated on Serines 13 and 16, having effects on Htt protein levels and aggregation. Changing these serines in a BACHD mouse model of HD prevents onset of disease in the mice, suggesting that modifying these residues could potentially alter disease progression and/or onset. Recent data also shows that phosphorylation and SUMO modification of Htt are co-regulated, together with other modifications that will be discussed. Therefore, understanding the underlying molecular machinery and functional consequences of specific post-translational modifications are emerging as potential 
therapeutic targets. Here, we will present data suggesting that specific SUMO-modifying enzymes and targeting of Htt phosphorylation events represent novel targets and approaches for treatment of HD.

11:20-11:30 AM

\section{LATE-BREAKING RESEARCH}

PlatForm PRESENTATION-Feasibility of a study of Coenzyme Q10 in pre-manifest Huntington's disease: The PREQUEL study.

K. Biglan, ${ }^{1}$ E. Julian-Baros, ${ }^{1}$ N. Yoritomo, ${ }^{2}$ and C. Ross ${ }^{2}$ for the Huntington Study Group PREQUEL Investigators. ${ }^{I}$ University of Rochester, Rochester, NY, USA and ${ }^{2}$ Johns Hopkins University, Baltimore, MD, USA.

Background: The PREQUEL study is evaluating the safety and tolerability of three dosages of Coenzyme Q10 (CoQ) in a population of expansion positive individuals pre-manifest (prior to motor diagnosis) for Huntington disease (HD). This study began enrollment in February 2010 and represents the first multi-center interventional study in this population.

Objective: We report on the preliminary feasibility of enrolling and retaining participants pre-manifest for HD in an interventional clinical trial and the preliminary blinded tolerability of CoQ in this population.

Methods: PREQUEL is a double blind 20 week parallel group multicenter trial with participants pre-manifest for HD randomized to receive $600,1,200$, or 2,400 mg/day of Coenzyme Q10. The primary outcome is tolerability defined by the ability to complete the 20 weeks of follow-up on the original treatment assignment. Secondary outcomes include changes in serum levels of 8-hydroxydeoxyguanosine, a measure of oxidative injury. Feasibility was assessed by evaluating enrollment activity and trends. Tolerability was assessed by evaluating withdrawals, drug suspensions and dosage reductions, and adverse events.

Results: As of July 28, 2010, 44 participants have enrolled at eight sites. Eighty-six percent of participants were referred by site staff, $9 \%$ by the HDSA, and $2 \%$ each from family/friend, geneticist, HDTrials.org, other neurologist. There have been no premature withdrawals, study drug suspensions or dosage reductions. There have been 30 adverse events in 28 participants, most commonly gastrointestinal $(n=9)$, and no serious adverse events.

Conclusion: Clinical trials in a population pre-manifest for HD are feasible. Preliminary data suggest that CoQ is well tolerated in this population.
11:30-11:40 AM

\section{LATE-BREAKING RESEARCH}

Platform PRESENTATION-A Randomized, Double-Blind Placebo-Controlled Trial of ACR16 in Huntington's Disease.

HART Investigators of the HSG (A. McGarry: corresponding author; K. Kieburtz: presenting author). University of Rochester, Rochester, NY, USA.

Background: Huntington's disease (HD) involves disrupted dopaminergic function in the basal ganglia, which translates into involuntary movement and loss of volitional movement over time. Aberrant dopaminergic regulation has also been implicated in cognitive and behavioral disturbances in HD. Compounds that reduce dopaminergic activity form the basis of current therapeutic strategies; however, the efficacy of treatments (i.e., dopamine receptor blockade, monoamine depletion) is suboptimal, particularly for voluntary movement. ACR16, a novel compound that can stabilize dopaminergic activity, produced a trend towards improved voluntary movement, anxiety, and depression when given to $58 \mathrm{HD}$ subjects in a previous 28-day trial. We sought to substantiate these observations in a larger trial.

Methods: In a randomized, double-blind parallel group design, we tested three doses of ACR16 (10 BID, 22.5 BID, 45 BID) and placebo over a 12-week treatment period. The primary outcome measure was change in voluntary motor function as measured by modified motor score, a subset of the UHDRS motor scale. Secondary objectives were to examine changes in clinical impression of change (CGI-C), total UHDRS behavioral assessment, total HADS score, Stroop Word Reading Test, and safety/tolerability. Exploratory analyses investigated effects of ACR16 on dysarthria, gait, Stroop interference test, Symbol Digit Modalities Test, verbal fluency, Trail-making A, the total UHDRS motor score, maximal chorea, HADS subscores, and UHDRS subscores.

Results: Two hundred twenty-seven HD subjects were recruited and enrolled from 29 North American sites over a 20-month period, with the last subject visit on August 9, 2010. The results are currently being collected and analyzed.

Conclusions: To follow shortly after data analysis.

11:40 AM-12:30 PM

PANEL DISCUSSION-Question and answer session between the day's speakers and the audience. 


\section{POSTER SESSION}

Posters will be staffed from 8:00-9:00 AM and 10:3010:50 AM in the Aventine Ballroom.

\section{POSTER 1}

\section{Assessing Patients' Access to Tetrabenazine (Xenazine) after FDA Approval.}

A. Palao, C. Hunter, G. Mostile, and J. Jankovic. Parkinson's Disease Center and Movement Disorders Clinic at Baylor College of Medicine, Houston, TX, USA.

Background: In 2008, the monoamine-depleting drug tetrabenazine (Xenazine) was approved by the FDA for the treatment of the chorea associated with Huntington disease (HD). The drug is now available as an orphan therapy through specialty pharmacies.

Objectives: To assess patient satisfaction with regard to current accessibility of the drug as compared to prior utilization through Investigational Exemption for a New Drug, granted to $\mathrm{JJ}$ in 1979.

Methods: A self-administered questionnaire was constructed to assess patients' experiences, costs, and attitudes related to perceived efficacy and tolerability of tetrabenazine prescribed for the treatment of a variety of hyperkinetic movement disorders. A mail survey was then conducted using as study population all patients currently enrolled in the tetrabenazine treatment program at the Baylor College of Medicine Parkinson's Disease Center and Movement Disorders Clinic (PDCMDC).

Results: The questionnaire was mailed to 265 patients and 240 (90.6\%) completed the survey. The respondents included 76 (31.7\%) patients with Tourette's syndrome, 87 (36.2\%) with tardive dyskinesia, and $42(17.5 \%)$ with HD. Tetrabenazine use for more than 5 years was reported by 95 (39.6\%) respondents. Prior to taking tetrabenazine, $220(91.7 \%)$ patients indicated their symptoms made them moderately to severely ill. Of the $235(97.9 \%)$ patients currently taking tetrabenazine, mean daily dose of $54.95 \pm 23.91 \mathrm{mg}$. Some side effects from tetrabenazine were reported by $135(56.2 \%)$ patients and included drowsiness (29.2\%), slowness of movement (14.6\%), restlessness (5.4\%), and depression or mood change $(12.5 \%)$. Before FDA approval, $192(80 \%)$ patients purchased the drug from the PDCMDC. With the current Specialty Pharmacy Program, 156 $(65 \%)$ patients were initially approved for coverage of tetrabenazine by their insurance carrier; most of the other claims were successfully appealed. Before FDA approval 137 $(57.1 \%)$ of patients were paying $\$ 200-\$ 300$ per bottle, but since the FDA approval $200(83.3 \%)$ have less than $\$ 100$ outof-pocket cost. Of the responders, 219 (91.2\%) patients stated that they were satisfied or very satisfied with their response to tetrabenazine and 205 (85.4\%) with the Specialty Pharmacy Program.

Conclusions: Our survey indicates a high level of satisfaction among patients prescribed tetrabenazine for their hyperkinetic movement disorder with cost-effective access to the drug via a specialty pharmacy system.

\section{POSTER 2}

The impact of isotype-selective histone deacetylase inhibitors on disease phenotypes in Huntington's disease animal models.
B. Tang, ${ }^{1}$ J. Pallos, ${ }^{2}$ H. Plasterer, ${ }^{3}$ J. Rusche,${ }^{3}$ J. Gottesfeld, ${ }^{1}$ J. Marsh, ${ }^{2}$ and E. Thomas. ${ }^{1}{ }^{1}$ Department of Molecular Biology, The Scripps Research Institute, La Jolla, CA, USA, ${ }^{2}$ Departments of Developmental and Cell Biology and Pathology, University of California, Irvine, CA, USA, and ${ }^{3}$ Repligen Corporation, Research and Development, Waltham, MA, USA.

We secured a grant from the Victorian Legal Services Board to fund a follow on project from RESPOND-HD, to analyze and explore the Australian experience of genetic discrimination (GD), using HD as a case model. The objectives are 1) improving knowledge of laws related to GD, 2) improved legal services, and 3) development of easily accessible resources (brochure, website) informing consumers of their rights.

Results: Sixty participants (65\% female; 35\% male; $60 \%$ HD gene positive; $40 \%$ gene negative), aged between 20 and 69 years. Thirty-two percent reported experiencing GD, with mean number of incidences $3.17( \pm 3.35)$. Participants experienced discrimination in employment, social, and insurance domains. For example, $17 \%$ of respondents had been refused insurance coverage, $20 \%$ offered coverage only at a higher premium, $55 \%$ told because of family history they could only get limited coverage, and $36 \%$ were told that due to test results they could only get limited coverage.

Awareness of Legislation. Victorian anti-discrimination laws are contained in the Equal Opportunity Act 1995, Disability Discrimination Act 1992, and Human Rights and Equal Opportunity Commission Act 1986. Seventeen percent of participants knew where to make a complaint regarding GD. Twenty-seven percent knew of laws preventing employers from unfairly using genetic information. Fifteen percent knew laws preventing health insurance companies from accessing or requiring genetic information about an insured person. Ten percent knew of laws preventing these companies from using genetic information to deny insurance. We aim to increase these percentages. We are funded to hold focus groups to discuss results and clarify issues with several cohorts (gene negative participants, gene positive participants, family members, case workers, health professionals, and researchers), and also for a public launch of the above-mentioned resources.

\section{POSTER 3}

What Were You Thinking? Individuals at Risk for Huntington Disease Talk About Having Children.

K.A. Quaid, ${ }^{1}$ M.M. Swenson, ${ }^{2}$ S.L. Sims, ${ }^{2}$ J.M. Harrison, ${ }^{3}$ C. Moskowitz, ${ }^{4}$ N. Stepanov, ${ }^{5}$ G.W. Suter, ${ }^{6}$ and B.J. Westphal ${ }^{7}$ for the Huntington Study Group PHAROS Investigators and Coordinators. 'Indiana University School of Medicine, Indianapolis, IN, USA, ${ }^{2}$ Indiana University School of Nursing, Indianapolis, IN, USA, ${ }^{3}$ Emory University School of Nursing, Atlanta, GA, USA, ${ }^{4}$ Columbia University, New York, NY, USA, ${ }^{5}$ Ohio State University, Dublin, OH, USA, ${ }^{6}$ Hereditary Neurological Disease Centre, Wichita, KS, USA, and ${ }^{7}$ Hennepin County Medical Center, Minneapolis MN, USA.

Most of the research on reproduction in those at risk for Huntington Disease (HD) has focused on the impact of genetic testing on reproductive decision-making. The goal has been to determine whether discovering one is a carrier of the HD mutation changes an individual's or couple's decision to start a 
family or to have more children. The purpose of this qualitative study was to examine reproductive decision-making in a sample of individuals at risk for HD who has chosen not to pursue genetic testing. Data were obtained from 55 individuals participating in the Prospective Huntington at Risk Observational Study (PHAROS). PHAROS is a multi-site study of 1,001 individuals at risk for HD who had chosen not to be tested. Interviews were conducted at six PHAROS sites across the country in Indianapolis, New York, Atlanta, Ohio, Wichita, and Minneapolis. Qualitative descriptive methods were used to explore reproductive decision making in three groups: 1) those who knew of their risk and decided to have children anyway (group 1); 2) those who did not know or were unclear about their risk when they had children (group 2); and 3) those who knew of their risk and decided not to have children (group 3).

In group 1, we identified four major themes; 1) hoping for a cure, 2) feeling guilty, 3) magical thinking, and 4) just another something. In group 2, we identified two major themes: 1) too little, too late and 2) getting it wrong. In group 3, we identified three major themes: 1) vigilant witness, 2) stopping HD, and 3) being alone.

In our discussion, we discuss these themes in the context of reproductive decision making. We also discuss the delicate balance healthcare professionals and genetic counselors must maintain between the benefits of providing hope for our patients and families and the dangers of offering unrealistic expectations about the time that scientific advances may realistically expect to materialize.

\section{POSTER 4}

Development of a health professionals webpage on the Movement Disorder Society webpage.

C. Moskowitz ${ }^{1}$ and M. Graziano. ${ }^{2}{ }^{1}$ Columbia University, New York, NY, USA and ${ }^{2}$ Private Practice, Esch-sur-Alzette, Luxembourg.

Objective: To build an international multidisciplinary interactive virtual group of professionals in counseling, nursing, rehabilitation, and genetics, who enrich and broaden the scope of care, management and research initiatives in movement disorders.

Background: From its inception, the society has openly welcomed membership and encouraged participation among non-physicians (Bylaws, 1985; Strategic Plan, 2006). Barriers to full membership and fuller participation are assumed to be geographic, financial, leadership, and program issues. Sessions and posters by non-physician faculty have increased in recent years. The Executive Committee of the MDS is more proactive. The Internet is now a robust educational tool beyond facilitating communication. The confluence of these factors has reduced barriers to full society participation.

Methods: Invited by the Executive Committee to design a Webpage on the MDS Website, we met to formulate goals and objectives and identify others with special interest and/or expertise in movement disorders. The European Huntington Disease Network and the Parkinson Study Group encouraged their colleagues to logon to the Website, learn about the society and the new Health Professionals group. The Society's project management tool will allow more colleagues to work collaboratively on multidisciplinary projects in cross-training (one discipline learning from another) and improving the quality and expanding the scope of care.

Results: Icons are linked to role descriptions for each rehabilitation therapy, nursing, genetics, dietetics/nutrition, and clinical psychology. One icon outlines the purpose and evolving nature of the group. A resource section highlights educational links. The 2010 needs survey will document demographics, culture of learning in country of practice, and the unmet educational needs of health professionals.

Conclusion: In our first year, we have documented a need to bring together movement disorder health professionals who work in various disciplines to learn from each other, address the unmet educational needs of their colleagues and disseminate new knowledge world-wide.

\section{POSTER 5}

Assessment of the Progression of Huntington's Disease during the Preclinical and Early Symptomatic Phases: a Multi-Imaging Longitudinal Study.

C. Tang, ${ }_{3}^{1}$ A. Feigin, ${ }^{1,2}$ N. Gerber, ${ }^{1}$ Y. Ma, ${ }^{1,2}$ S. Peng, ${ }^{1}$ M. Guttman, ${ }^{3}$ J. Paulsen, ${ }^{4}$ V. Dhawan, ${ }^{1,2}$ and D. Eidelberg. ${ }^{1,2}$ ${ }^{1}$ Center for Neurosciences, The Feinstein Institute for Medical Research, North Shore-Long Island Jewish Health System, Manhasset, NY, USA, ${ }^{2}$ Department of Neurology, North Shore University Hospital and New York University School of Medicine, New York, NY, USA, ${ }^{3}$ Department of Neurology, University of Toronto, Toronto, Canada, and ${ }^{4}$ Department of Neurology, University of Iowa, Des Moines, IA, USA.

Objective: To examine longitudinal changes in brain metabolism, doperminergic function, and volume in presymptomatic Huntington's disease (pHD) and early symptomatic HD (sHD) patients.

Methods: Twelve pHD gene mutation carriers [age: $46.8 \pm$ 11.0 years; CAG repeat length, $41.6 \pm 1.7$; estimated years-toonset, 10.3 \pm 8.6$]$ underwent serial PET imaging with $\left[{ }^{18} \mathrm{~F}\right]-$ fluorodeoxyglucose (FDG) and ${ }^{11} \mathrm{C}$-raclopride (RAC), MRI, and clinical assessments at baseline, 1.5, 4, and 7 years. Four pHD subjects became symptomatic by 4 years of follow-up. Additionally, five new sHD patients (disease duration: $3.0 \pm$ 0.71 years) were scanned once. Longitudinal FDG-PET data were analyzed using a within-subject spatial covariance Ordinal-Trends (OrT) model to identify a specific metabolic pattern associated with preclinical HD progression. Longitudinal RAC-PET data were analyzed using region-of-interest analysis to examine changes in striatal $\mathrm{D}_{2}$ receptor binding. Longitudinal MRI data were analyzed using voxel-based morphometric SPM analysis to examine changes in volume.

Results: Longitudinal FDG-PET revealed a metabolic network characterized by progressively decreasing metabolism in striatal, thalamic, insular, prefrontal, cingulate and occipital areas, and concurrent metabolic increases in cerebellar, pontine, orbitofrontal, and temporal regions. Expression of this HD-OrT pattern increased $(p<0.001)$ in individual $\mathrm{pHD}$ subjects over time, and was elevated $(p<0.001)$ in the sHD patients. RACPET showed linear decreases in caudate and putamen $D_{2}$ receptor binding over time. MRI showed a longitudinal loss of volume $(p<0.001)$ in caudate, prefrontal, cerebellar, temporal, insular, occipital, parahippocampal, primary somatosensory, and precuneus regions. Increased HD-OrT pattern expression in the pHD and sHD subjects remained significant after regional volumetric loss was corrected in the same subjects. The HDOrT pattern expression progressed at a faster rate $(p<0.001)$ than the striatal $D_{2}$ receptor binding and volume loss in the pHD subjects.

Conclusion: The HD-OrT metabolic network is a sensitive imaging biomarker of disease progression in preclinical and 
early symptomatic HD. Future studies to assess the reproducibility and validity of this measure are warranted.

\section{POSTER 6}

Influence of Dual-task performance on gait in Pre-manifest Huntington's disease.

A. Rao and K. Marder. Columbia University Medical Center, New York, NY, USA.

Background: Motor and cognitive impairments are detected well before clinical diagnosis of Huntington's disease (HD). However, the interaction of cognitive and motor task performance in pre-manifest HD is not known. We examined the influence of motor and cognitive dual task on gait.

Methods: Pre-manifest HD subjects and healthy control subjects, matched for age and gender, were tested under three conditions: 1) walking at preferred speed (P); 2) performing manual task while walking (M); and 3) performing a verbal fluency task while walking (C). Spatio-temporal variables were measured using the GAITRite ${ }^{\circledR}$ mat $(4.6 \mathrm{~m}$ long) at $80 \mathrm{~Hz}$. We analyzed measures related to gait speed, dynamic balance, and temporal patterning (stride-to-stride variability).

Results: Measures related to speed (velocity and stride length) decreased whereas step time increased progressively from preferred to manual to cognitive condition for both groups of subjects. Neither manual nor cognitive tasks had an influence on variables related to dynamic balance (support base and double support percent). Stride-to-stride variability (stride length $\mathrm{CV}$ and swing time $\mathrm{CV}$ ) were higher for the cognitive dual task in premanifest HD subjects compared with control subjects.

Discussion: Pre-manifest HD subjects demonstrated impairments in gait speed and stride-to-stride variability compared with healthy controls. Cognitive dual task demonstrated greater interference on gait in pre-manifest HD subjects. Dual task gait paradigm may be an effective probe to detect subtle gait impairments in pre-manifest HD and stride-to-stride variability, in particular, may be a good marker of disease onset and progression in HD.

\section{POSTER 7}

Prominent psychiatric, behavioral, and cognitive manifestations in Australian Huntington Disease patients with Maltese heritage.

E. McCusker, ${ }^{1}$ A. Lownie, ${ }^{1}$ and C. Loy. ${ }^{1,2}{ }^{1}$ Neurology Department, Westmead Hospital, Sydney West Area Health Service, Sydney, NSW Australia and ${ }^{2}$ School of Public Health, University of Sydney, Sydney, Australia.

Huntington Disease (HD) may have variable manifestations. Psychiatric, behavioral and cognitive changes may precede motor onset. Onset of HD is most often defined as motor onset with choreiform movements. To date, no modifier has been identified in patients with predominantly psychiatric, behavioral and cognitive manifestations. Faull et al. reported neuropathological differences in patients whose family members noted mood dysfunction as the main feature.

Australians with Maltese heritage make up the largest nonAnglo Saxon group seen by the Huntington Disease Service in Sydney Australia. The clinical impression is that these patients are more likely to have prominent psychiatric, behavioral and cognitive presentations, and manifestations during the course of the disease.
Aim: To review mode of presentation and history of psychiatric, behavioral, and cognitive impairment in Australian Maltese HD patients known to the HD Service.

Method: Review of Service database between 1995 and 2010.

Results: Twenty-three HD patients with Maltese heritage were identified. Of these, 17 had significant behavioral, psychiatric, and cognitive manifestations at times requiring prolonged inpatient care, some with relative preservation of motor function late into the disease course.

Maltese carrying the HD mutation who immigrated to Australia may have a gene modifier or a disease type with more prominent non-motor manifestations. These kindreds, as well as those on the island of Malta in the Mediterranean, may be a population for future study of gene modifiers and for a founder effect.

\section{POSTER 8}

Successful Recruiting Strategies at Evergreen Neuroscience Institute, a new Huntington Study Group Investigative Site. L. Goodman, ${ }^{1}$ C. Sia, ${ }^{2}$ and P. Agarwal. ${ }^{2}{ }^{1}$ Huntington's Disease Drug Works, Lake Forest Park, WA, USA and ${ }^{2}$ Evergreen Neuroscience Institute, Kirkland, WA, USA.

Objective: To determine the effect of community grass roots efforts on recruitment of Huntington's (HD) participants to HART at Evergreen Neuroscience Institute, a new Huntington Study Group (HSG) investigative site lacking an HD patient base.

Background: Evergreen Neuroscience Institute in Kirkland, Washington signed on as a new HSG investigative center in late summer of 2009 and subsequently recruited for HART during the 8 months prior to full enrollment. Evergreen has historically served a Parkinson's disease population at the Booth Gardner Parkinson's Care Center, but had no base of patients seen for HD.

Methods: Referral sources, rate of recruitment, and distance traveled for each participant was tracked between August 2009 and April 2010.

Results: Seventeen potential participants were screened with 14 meeting criteria for enrollment. Fourteen completed the trial, (with one failing the safety follow-up visit) which was third highest among HSG sites. In addition, rate of enrollment at Evergreen was highly efficient with five individuals lined up for site contact on the first day of enrollment. Sources of referral were multiple and included grass-root efforts in support groups and their family contacts (8/17), HD advocacy websites (2/17), HSG website (2/17), HDSA/HDTrials websites (2/17), PCP referral (1/17), and self-referral (1/17). The average distance traveled by participants to Evergreen was 230 miles, ranging from 5 to 2,400 miles.

Conclusions: Grass root and web-based recruitment strategies were successful for efficient recruitment of a significant number of participants to this site that could not draw from an existing HD patient base. This type of recruitment effort is increasingly vital to augment traditional Center of Excellence and HSG site population efforts. It is increasingly important that Huntington's organizations encourage, train, support, and incorporate grass roots efforts as part of recruitment strategy.

\section{POSTER 9}

Multimodal approach to seeking biomarkers in Huntington disease by MRI and PET scanning.

F. Squitieri, ${ }^{1}$ S. Orobello, ${ }^{1}$ U. Sabatini, ${ }^{2}$ A. Cherubini, ${ }^{2}$ and A. Ciarmiello. ${ }^{3}$ Neurogenetics Unit, IRCCS Neuromed and 
Centre for Rare Diseases, Pozzilli, Italy, ${ }^{2}$ IRCCS Santa Lucia Foundation, Rome, Italy, and ${ }^{3}$ Unit of Nuclear Medicine, S. Andrea Hospital, La Spezia, Italy.

Multimodal methodological approaches taking in account early changes in structure and metabolism of brain areas represent a key procedure to highlight potential biomarkers of progression of Huntington disease (HD) pathology since the presymptomatic life stages. We evaluated, by 3DT1-weighted images MRI, the macrostructure, microstructure and iron content of five vulnerable subcortical structures (high risk: caudate, putamen and pallidus; and low-risk: thalamus and accumbens) in comparison with medial temporal lobe structures (hippocampus and amygdala) relatively preserved in early stages of the disease. Seventeen presymptomatic and 14 symptomatic (stages I-II) and 31 age- and gender-matched control subjects were scanned using the same protocol. We also studied a cohort of 43 gene positive subjects with 18-fluoro-deoxy-glucose positron emission tomography (PET) scan and tested whether peripheral blood levels of transforming growth factor betal (TGF-beta1) could be associated with metabolic changes in caudate. By MRI, pre-HD subjects had a selective decrease of volume and microstructural changes in high-risk structures (caudate, putamen, and pallidus) and increased iron accumulation in all three nuclei. HD patients had lower volume than preHD in all high-risk nuclei, higher microstructural impairment in the striatum, but not significant differences in iron content. The lesion extended to low-risk subcortical nuclei (thalamus and accumbens). Furthermore, volume and microstructure correlated with the clinical measures of progression of the disease. By PET scan, we observed significantly higher glucose metabolism in far-from-onset group (asymptomatic) subjects vs. those nearto-onset (premanifest) and vs. manifest-HD group $(P<0.0001)$ and a slight but significant difference in glucose metabolism between premanifest vs. manifest-HD group $(p<0.05)$. We identified a cut-off value of caudate glucose metabolism between asymptomatic and premanifest subjects and serum TGF-beat1 levels decreased linearly in pre-HD subjects with decreasing caudate glucose uptake. Combination of multimodal MRI and FDG-PET scans with peripheral biological changes may represent the methodology to seeking HD biomarkers.

\section{POSTER 10}

Low levels of TGF beta 1 in Huntington disease brain and peripheral tissues: could it be a new possible biomarker? A. Silvia, ${ }^{1}$ S. Orobello, ${ }^{1}$ T. Martino, ${ }^{1}$ A.M. Griguoli, ${ }^{1}$ E. Amico, ${ }^{1}$ G. Battaglia, ${ }^{2}$ F. Nicoletti, ${ }^{2}$ and F. Squitieri. ${ }^{1}{ }^{1}$ Neurogenetics Unit and Rare Disease Centre, IRCCS Neuromed, Pozzilli (IS), Italy and ${ }^{2}$ Neuropharmacology Unit, IRCCS Neuromed, Pozzilli (IS), Italy.

Huntington Disease (HD) is a neurodegenerative disorder caused by a CAG expansion mutation in huntingtin (htt). Mutant htt induces cell death modulating several molecular pathways, including cell signaling, transcriptional machineries, and neurotrophins production and release. Recently, we have reported an early defect in TGFbetal production in HD subjects and in two HD mouse models (R6/2 and YAC128). TGFbeta1 is a pleiotropic cytokine with an established neuroprotective function as well as a powerful anti-inflammatory role. Since TGFbetal is predominantly expressed in the immune system and since myeloid-derived immune cells (i.e., glial cells) are implicated in neurodegeneration, we investigated the levels of this cytokine in immortalized human astroglial cell lines expressing wild-type (SVGp12-25Q) and mutant (SVGp12-72Q) Htt-exon1, and in macrophages from HD subjects and R6/2 mice. We analyzed the levels of TGFbeta1 within cell lines by flow cytometry; and by quantitative real time (RT)-PCR. All subjects HD $(n=28)$ were clinically and genetically evaluated and age matched with health controls $(n=$ 14). Our analysis showed significant reduced levels of TGFbetal in human and mouse HD macrophages, and in human immortalized HD glial, compared to wild-type, cells. Interestingly, TGFbeta1 levels in human macrophages depended on the disease stage. Such scenario was confirmed by experiments in HD mice that showed significant low levels of the cytokine at the presymptomatic stage. While confirming a TGFbeta1 related dysfunction in peripheral and brain tissues in HD, we remark the potentiality for searching novel possible progression related markers in HD.

\section{POSTER 11}

The Effect of Video Game-Based Exercise on Dynamic Balance, Mobility and UHDRS Neuropsychiatric Test Scores in Individuals with Huntington's Disease.

A. Kloos, ${ }^{1}$ N. Fritz, ${ }^{1}$ S. Kostyk, ${ }^{2}$ and D. Kegelmeyer. ${ }^{1}{ }^{1}$ The Ohio State University, Department of Physical Therapy, Columbus, OH, USA and ${ }^{2}$ The Ohio State University, Department of Neurology, Columbus, OH, USA.

Please see Platform Presentation above for abstract body.

\section{POSTER 12}

A Comparison of Tetrabenazine and Olanzapine in the Management of Chorea in Patients with Huntington's Disease.

T. Tempkin, V. Wheelock, and R. Pauli. University of California Davis Health System, Department of Neurology, Sacramento, CA, USA.

Chorea is a common motor feature in Huntington's disease. Severity ranges from mild chorea in isolated limbs, to severe movement involving all limbs, trunk, head, and neck. Moderate and severe chorea can interfere with a patient's functional status and threaten mobility and independence. Milder chorea is often distressing and socially embarrassing

In November 2008, Tetrabenazine came to market as the first medication approved in the USA by the FDA for treatment of chorea in Huntington's Disease. Prior to this time, clinicians would often use medications "off label".

Anti-psychotic medications such as Olanzepine have shown modest improvement in chorea (Wheelock, et al., Neurotheraputics, April 2008, pg 365). Side effects of weight gain, metabolic changes, and sedation are well known in this classification of medication and can be a barrier to treatment of chorea.

We did a retrospective comparative analysis of 55 patients seen in the Huntington's disease clinic from January 2009 to June 2010 at the HDSA Center of Excellence, University of California Davis. Of the 55 reviewed, 29 had chorea scores prior to initiation of anti-chorea therapy and after starting treatment. Treatment groups included seven patients on Tetrabenazine alone, 19 patients on Olanzepine alone, and three patients on both agents. The Olanzepine monotherapy group showed a $26 \%$ improvement in chorea scores $(p=$ 0.0193 ), and the Tetrabenazine monotherapy group showed a $40 \%$ improvement in chorea scores $(p=0.00289)$. Of the three patients on combination therapy, all had started treatment 
initially with Olanzepine, with Tetrabenazine added later. Mean chorea scores in this group improved $6.7 \%$ after the addition of Tetrabenazine.

Conclusion: Both agents, Olanzepine and Tetrabenazine were effective in the management of chorea, with Tetrabenazine showing greater improvement in chorea scores. Combination therapy showed modest improvement after adding Tetrabenazine to previously established therapy with Olanzepine. This retrospective review is limited by sample size and unequal distribution between treatment groups. A prospective study is recommended.

\section{POSTER 13}

Variability in age of onset in Huntington disease: a new model.

J. Pillai, ${ }^{1}$ S. Edland, ${ }^{2,3}$ J. Goldstein, ${ }^{3}$ S. Gluhm, ${ }^{4}$ G. Peavy, ${ }^{3}$ and J. Corey-Bloom. ${ }^{1}{ }^{1}$ VA San Diego Medical Center, Department of Neurology, San Diego, CA, USA, ${ }^{2}$ University of California, San Diego, Department of Statistics, UCSD Shiley-Marcos Alzheimer's Disease Research Center, La Jolla, CA, USA, ${ }^{3}$ University of California, San Diego, Department of Neurosciences, UCSD Shiley-Marcos Alzheimer's Disease Research Center, La Jolla, CA, USA, and ${ }^{4}$ Department of Psychology, San Diego State University, San Diego, CA, USA.

Variability in age of disease onset for given numbers of CAG trinucleotide repeats in Huntington disease

Huntington disease (HD) has prompted many investigators to look for genetic, environmental, or other clinical modifiers.

The predominant model for disease onset is the single hit hypothesis; however, direct evidence that clinical onset is consistent with this is lacking. We therefore elected to examine the best model for explaining the rate of clinical onset of disease using a well-characterized population of HD subjects at one academic medical center. One hundred fifty HD patients (ages 18-75 years), with CAG repeats between 40 and 51, were analyzed.

In each repeat number, the age of onset was plotted against the fraction of cases yet to be diagnosed. We found that Gompertz function, rather than a first-order exponential of the single hit model, best fit the data with regard to age at onset in HD. Gompertz function models a neuronal death process in which the clinical expression of disease is influenced by the linear decrease in caudate volume with age. As such, Gompertz function may be the best model for explaining the variability in range of disease onset in HD.

\section{POSTER 14}

Clinical severity of Huntington's disease does not always correlate with neuropathologic stage.

J. Pillai, ${ }^{1}$ L. Hansen, ${ }^{2}$ E. Masliah, ${ }^{2}$ J. Goldstein, ${ }^{2}$ S. Edland, ${ }^{2,3}$ and J. Corey-Bloom. ${ }^{1}$ VA San Diego Medical Center, Department of Neurology, San Diego, CA, USA, ${ }^{2}$ University of California, San Diego, Department of Neurosciences, UCSD Shiley-Marcos Alzheimer's Disease Research Center, La Jolla, CA, USA, ${ }^{3}$ University of California, San Diego, Department of Statistics, UCSD Shiley-Marcos Alzheimer's Disease Research Center, La Jolla, CA, USA, and ${ }^{4}$ Department of Psychology, San Diego State University, San Diego, CA, USA.

Objective: To assess whether clinical disease severity in HD correlates with pathologic burden at autopsy.
Background: Postmortem grading or staging of neuropathological severity in HD, as proposed by Vonsattel, follows an ordered pattern of involvement that includes first the tail of the caudate nucleus, its body and head, the putamen, and finally the globus pallidus and possibly neocortex. The grading system has five grades $(0-4)$ of severity of striatal involvement. The presumption is that clinical disease severity in HD reflects neuropathologic staging.

Methods: This is an autopsy series of 24 well-characterized HD patients from one academic medical center. Most subjects were followed closely through our HDSA Center of Excellence and received extensive neurologic evaluation, including cognitive testing, as part of their ongoing evaluations. Autopsy was performed within $24 \mathrm{~h}$ of death using a standardized protocol. Pathologic assessment was made by one observer (L.A.H.). Subjects were stratified on the basis of Vonsattel staging as mild/moderate (stage $1-2, n=7$ ) or severe (stage 3-4, $n=17$ ).

Results: There were ten males and 14 females, with ages at death that ranged from 35 to 83 years, included in this analysis. Despite similar mean durations of disease, the subjects classified pathologically as severe were significantly younger, had higher CAG repeats, and lower fixed brain weights than those classified as mild/moderate (all $p<0.05$ ). In spite of this, mean MMSE scores prior to death (23.7 vs. $24.0, \mathrm{NS})$ and mean intervals from last testing to death ( 2.1 vs. 2.7 years, NS) were surprisingly similar between the two groups.

Conclusions: These results suggest that the extent of striatal changes in HD may not always correlate with clinical disease severity. Further studies will be needed to confirm and extend these findings.

Study supported by: UCSD Huntington's Disease Society of America Center of Excellence; UCSD Shiley-Marcos Alzheimer's Disease Research Center NIH P50 AG 005131 and NIH RO3 AG 034439 (S.D.E.)

\section{POSTER 15}

\section{Depression in Prodromal Huntington Disease.}

E.A. Epping, J. Mills, M.M. Smith, L.J. Beglinger, K.C. Rowe, J.G. Fiedorowicz, J.S. Paulsen, and the PREDICT-HD Investigators of the Huntington Study Group. The University of Iowa, Iowa City, IA, USA.

Background: The PREDICT-HD study is prospectively following individuals with the mutation for Huntington disease prior to onset of motor symptoms. This project aims to characterize the symptoms of depression and treatment in the prodromal phase of HD.

Methods: Eight hundred two individuals with prodromal HD (prHD) were evaluated at their initial visit in the PREDICT-HD study for symptoms of depression using the Beck Depression Inventory II (BDI-II), UHDRS Behavioral Scale, and depression subscale of the Symptom Checklist 90 Revised (SCL90R). Antidepressant treatment at the first visit was reported. Among those with prHD and at least mild symptoms on the BDI-II, a factor analysis of questions across all three assessments was completed to identify the most significant domains of depressive symptoms in this population. Longitudinal changes were also evaluated between visits at baseline and year 3 .

Results: Based on standard BDI-II cutoffs, $22 \%$ reported depressive symptoms of mild or greater severity. The prevalence of antidepressant use varied by minimal (17\%), mild 
$(36 \%)$, moderate $(38 \%)$, or severe $(56 \%)$ BDI-II symptoms. The factor analysis identified eight separate domains of symptoms encompassing questions across all assessments, with the most significant factors related to self-critical thinking, physical symptoms, and irritability/agitation/anxiety. Longitudinal analyses did not show any significant changes in symptoms over time.

Summary: Depressive symptoms were present in a minority of prHD subjects, and many with more severe symptoms did not report antidepressant use. Results of the factor analysis may assist in identifying the most significant symptoms of depression in this population. The lack of longitudinal changes in symptoms may indicate depression does not worsen with progression of HD; however, more study is needed to identify relationships between depression, treatment, and progression of other prHD symptoms.

\section{POSTER 16}

Prescription Usage for Treatment Of Irritability, Perseverative Behaviors, and Chorea In Huntington's Disease.

M. Groves, ${ }^{1}$ E. van Duijn, ${ }^{2}$ D. Craufurd, ${ }^{3}$ K. Anderson, ${ }^{4}$ M. Guttman, ${ }^{5}$ E. Wexler, ${ }^{6}$ S. Perlman, ${ }^{6}$ A. Rosenblatt, ${ }^{7}$ D. van Kammen, ${ }^{8}$ J. Giuliano, ${ }^{8}$ J.-M. Burgunder, ${ }^{9}$ N. Goodman, ${ }^{10,11}$ and L. Goodman. ${ }^{11}{ }^{1}$ Beth Israel Medical Center, Psychiatry and Neurology, New York, NY, USA, ${ }^{2}$ Leiden University Medical Center, Psychiatry, Leiden, Netherlands, ${ }^{3}$ University of Manchester, Genetic Medicine, Manchester, UK, ${ }^{4}$ University of Maryland, Baltimore, MD, USA, ${ }^{5}$ University of Toronto, Toronto, Ontario, Canada, ${ }^{6}$ University of California-Los Angeles, Los Angeles, CA, USA, ${ }^{7}$ Johns Hopkins University, Department of Psychiatry, Baltimore, MD, USA, ${ }^{8}$ Cure Huntington's Disease Initiative (CHDI), Princeton, NJ, USA, ${ }^{9}$ Neurologische Klinik und Poliklinik, Universitätsspital Bern, Switzerland, ${ }^{10}$ Institute for Systems Biology, Seattle, WA, USA, and "llHuntington's Disease Drug Works, Seattle, WA, USA.

Introduction: Despite large gaps in evidence-based knowledge, clinical experience supports the use of pharmacologic treatments for many symptoms of Huntington's disease (HD). The project goal is to develop consensus guidelines based on expert clinical experience to improve quality of HD care.

Methods: The survey was developed by nine international experts, and designed as a highly iterative and systematic method of soliciting expert opinions on the pharmacologic treatment of irritability, perseverative behaviors, and chorea in HD. Fifty-five experts from Australia, Europe, and North America responded to at least one of the surveys.

Results: For irritability, SSRI was first choice of $56 \%$, and antipsychotic was first choice for $21 \%$, a mood stabilizing anticonvulsant $14 \%$, and benzodiazepine $2 \%$. For perseverative behaviors, SSRI was first choice of $75 \%$, an antipsychotic choice of $4 \%$, a mood stabilizing anticonvulsant choice of $6 \%$, and clomipramine $2 \%$. The remaining $13 \%$ chose to qualify the response to include two first choices. For chorea, an antipsychotic was first choice for $56 \%$, tetrabenazine $15 \%$, amantadine $6 \%$, and benzodiazepines $4 \%$. The remaining $13 \%$ chose to qualify the response to include two first choices. Drug choice for use as adjunctive therapy was widely variable.

Conclusion: Many areas of variability in treatment for irritability, perseverative behaviors, and chorea in HD, have been identified. The results of this survey will be used to create preliminary treatment algorithms in HD to improve patient care; such algorithms will then be refined based on the results of future clinical trials.

\section{POSTER 17}

Tracking Cognitive Change in Huntington's disease with the Mattis Dementia Rating Scale.

J. Corey-Bloom, S. Edland, J. Goldstein, G. Peavy, M. Jacobson, S. Lessig, and S. Gluhm. University of California, San Diego, La Jolla CA, USA.

Objective: To examine the usefulness of the Mattis Dementia Rating Scale (DRS) for tracking cognitive change in Huntington's disease (HD).

Background: Appropriate outcome measures are needed for clinical trials of therapeutic agents for cognition in HD. The Mattis DRS is a relatively brief dementia-screening instrument that may be sensitive to executive dysfunction. Although recently used to assess efficacy in a cholinesterase inhibitor trial in dementia associated with Parkinson's disease (Dujardin et al., 2006), little is known about its rate of decline in HD.

Design/Methods: We used a mixed effects model to analyze DRS scores obtained during 5 years of follow-up of 145 subjects with HD.

Results: Mean follow-up time was 2.8 (1.3 SD)years. Mean age of the subjects was 46.0 (11.9 SD)years, mean education was 13.9 (2.8 SD) years, and mean number of CAG repeats was 45.0 (4.5 SD). Mean rate of decline on the DRS was 3.2 (3.5 SD) points/year for the whole group, but 3.9 (2.7 SD) points/year for those with baseline MMSE $<26$. Using the DRS for cognitive trials in $\mathrm{HD}$, the sample size required to detect a clinically significant $50 \%$ slowing of decline in subjects with baseline MMSE <26 would be 104 patients/arm.

Conclusions/Relevance: The Mattis DRS declines significantly over time in an unselected HD population. Because of its sensitivity to executive dysfunction and ease of administration, the Mattis DRS may be a useful efficacy measure for therapeutic agents for cognition in HD.

Study supported by: UCSD Huntington's Disease Society of America Center of Excellence; UCSD Shiley-Marcos Alzheimer's Disease Research Center NIH P50 AG 005131 and NIH RO3 AG 034439 (S.D.E.)

\section{POSTER 18}

18F-FDDNP PET Imaging in Huntington Disease.

F.C. Thiede, ${ }^{1}$ Y. Bordelon, ${ }^{1}$ V. Kepe ${ }^{2}$ S. Singh, ${ }^{1}$ J. Barrio, ${ }^{2}$ and J. Mazziotta. ${ }^{1}$ I Department of Neurology, University of California Los Angeles, Los Angeles, CA, USA and ${ }^{2}$ Molecular and Medical Pharmacology, David Geffen School of Medicine at UCLA, Los Angeles, CA, USA.

Biomarkers of underlying pathological processes are essential to expedite clinical research for therapeutic interventions in Huntington Disease. Dr. Barrio and colleagues developed the positron emission tomography (PET) amyloid molecular imaging probe 2-(1-\{6-[(2-[F18]fluoroethyl) (methyl) amino]2-naphthyl $\}$ ethylidene) malononitrile or [18F]FDDNP. This ligand has been shown to label pathology in living patients with neurodegenerative diseases characterized by intracellular and extracellular accumulation of amyloid or amyloid-like proteins including, Alzheimer disease and prion disease. We previously conducted a pilot study of five HD patients revealing that $[18 \mathrm{~F}] \mathrm{FDDNP}$ uptake was present in patients 
with premanifest and manifest disease. We have expanded our work to further explore the utility of [18F]FDDNP as an HD biomarker. Sixteen subjects with HD underwent [18F]FDDNPPET scanning including five premanifest subjects, three mild HD, six moderate HD, and two severe HD subjects. Parametric images of relative distribution volume (DVR) were generated by Logan graphical analysis with cerebellar gray matter as the reference region. Regions of interest were quantified on individual motion corrected DVR parametric images that were spatially normalized to a common brain space. Results revealed that striatal uptake of this ligand increased with disease progression. Mean striatal DVR was $1.33 \pm 0.12$ in the premanifest group, $1.37 \pm 0.1$ in the mild HD group, $1.45 \pm$ 0.13 in the moderate HD group, and $1.42 \pm 0.04$ in the severe HD group. Mild increased uptake of [18F]FDDNP was also present in frontal cortex in subjects with more advanced disease. This study provides evidence for the utilization of [F18]FDDNP-PET as a potential biomarker of the underlying pathologic substrate of disease in HD. Establishing an HD biomarker available for use in clinical trials would have great advantages in streamlining the translational research pathway.

Support: Hereditary Disease Foundation, Taube Foundation, Hogg Foundation, NIH

\section{POSTER 19}

Treatment of HD Chorea with Tetrabenazine in the Presence of Neuroleptics and Antidepressants.

B. Scott. Duke University Movement Disorders Clinic, Durham, NC, USA.

Background: Tetrabenazine (TBZ) is a dopamine-depleting drug which is FDA approved for treatment of chorea in Huntington's disease (HD). Out of the Duke University Movement Disorders Clinic population, seven HD patients were identified who had been taking TBZ in combination with either a neuroleptic medication, an antidepressant, or both.

The demographics of the seven HD patients were as follows: three females, four males; five Caucasians, one AfricanAmerican, one American Indian; age range 33-59 years (47士 9 s.d.). The dosing of TBZ ranged from 25 to $100 \mathrm{mg}$ daily in divided doses (52 $\pm 27 \mathrm{mgSD})$.

Methods: The neuroleptics used in combination with TBZ were: quetiapine in two points (25 and $400 \mathrm{mg}$ daily, respectively, tapered to $100 \mathrm{mg}$ daily in the latter case), olanzapine in one patient $(5 \mathrm{mg}$ daily, followed by stopping olanzapine after reaching a dosage of $25 \mathrm{mg}$ TBZ daily), risperidone in one patient $(2 \mathrm{mg}$ daily, tapered to 0 over 2 weeks), and aripiprazole in one patient (10 mg daily, stopped after titrated up to $50 \mathrm{mg}$ TBZ daily).

The antidepressants used in combination with TBZ among the seven patients were: sertraline in three points (range 50$200 \mathrm{mg}$ daily), amitriptyline in two points (20 and $30 \mathrm{mg}$ qHS, including $1 \mathrm{pt}$ taking both sertraline $75 \mathrm{mg}$ daily and amitriptyline $20 \mathrm{mg}$ nightly), fluoxetine in one patient $(40 \mathrm{mg}$ daily) and oxcarbazepine in one patient (150 mg daily).

Results: Patients were assessed in clinic as maintaining mild to moderate improvement of their chorea on TBZ (score of $1.6 \pm$ 1.1 SD where $0=$ none, $1=$ mild, $2=$ moderate, $3=$ marked, and 4 $=$ complete improvement of chorea) for an average duration of treatment of 16 months (range 1-34 months, $16 \pm 12$ months $\mathrm{SD})$. Of the seven HD patients, one experienced new-onset suicidal ideation without suicide attempts while taking a combination of TBZ $25 \mathrm{mg}$ and quetiapine $25 \mathrm{mg}$. This suicidality resolved off of TBZ and on increased quetiapine. A second patient with a previous history of two suicide gestures prior to taking TBZ was able to tolerate TBZ without developing additional suicidal gestures during 19 months of TBZ use.

Conclusions: These observations suggest that chorea in selected HD patients can be treated with TBZ in the presence of neuroleptic and antidepressant medications. Patients taking TBZ must be monitored for potential side effects of the drug including worsening depression and suicidal ideations/attempts.

Dr. Scott has received grant support from Lundbeck, Inc. and is a member of the Lundbeck speakers bureau.

\section{POSTER 20}

Caffeine Intake and Disease Characteristics in a Huntington's Disease Clinic Population.

A. Seward, ${ }^{1}$ C. Longwell III, ${ }^{1}$ G. Young, ${ }^{2}$ and S Kostyk. ${ }^{1}$ ${ }^{I}$ Department of Neurology, The Ohio State University, OH, USA and ${ }^{2}$ Center for Biostatistics, The Ohio State University, Columbus, OH, USA.

Several studies have suggested a possible neuroprotective effect of caffeine in a variety of neurodegenerative diseases including Parkinson's and Alzheimer's diseases. It has recently been suggested that caffeine use in Huntington's disease may be associated with earlier age of onset. In our clinic, we noted that caffeine intake seemed to be higher in patients with younger age of onset and more aggressive disease progression. To explore the relationship between caffeine consumption and HD in our clinic population, we performed a chart review over a 4 -year period. Approximately $90 \%$ of patients $(n=208)$ reported some caffeine intake during routine interviews. For the purposes of this study, the patients were separated into four groups, those whose daily caffeine intake was less than one caffeinated beverage a day $(22.6 \%)$, one to two beverages a day $(23.08 \%)$, three to five beverages a day $(24.04 \%)$, and more than five a day (19.23\%). Regression analysis was performed to study the relationship between caffeine intake and age of symptom onset and rate of disease progression. Age of onset was slightly later and motor progression slightly slower in patients who reported a moderate caffeine intake (one to two caffeinated beverages a day) compared with those who drank less than one caffeinated beverage a day or more than three caffeinated beverages a day. The highest level of caffeine intake was seen in those with earlier disease onset and a higher rate of progression. The results of the analysis of this retrospective chart review did not reach statistical significance; however, the trends may indicate the complex nature of the relationship of caffeine on HD disease presentation. While low level caffeine intake may have neuroprotective effects in $\mathrm{HD}$, moderate to high caffeine intake levels may contribute, possibly through different mechanisms of action, to earlier age of onset and/or more aggressive rates of disease progression.

\section{POSTER 21}

Computer simulations of striatal atrophy confirm age at onset of Huntington's disease is a valid phenotypic endpoint for genetic and epidemiologic studies.

S. Edland and J. Pillai. University of California San Diego, La Jolla, CA, USA.

Objective: To investigate the supposition that variable age at onset of Huntington's disease (HD) is explained by uninfor- 
mative stochastic variability in the timing of neuronal loss within the striatum, and to explore alternative models of striatal loss and symptomatic onset of disease.

Background: Little is known of the factors beyond polyglutamine repeat length that influence age at onset of HD. Cajavec et al. [Neurogenetics (2006) 7: 21-25] speculated that the stochastic nature of neuronal cell loss under the one-hit model of neurodegeneration in HD could explain a significant portion of the variability in age at onset of disease.

Methods: Following Cajavec et al., we simulated striatal neuron loss in HD as a random exponential process and assumed onset of disease occurred at the age at which the fraction of surviving striatal neurons reached a predetermined critical level.

Results: In computer simulations, stochastic neuronal cell loss contributed essentially no variability to the age at which a critical fraction of neurons within the striatum was lost. On the other hand, introducing random, half normally distributed person to person variability in the underlying exponential rate of cell loss and random, symmetric normally distributed variability in the fraction of striatal loss required to induce symptomatic disease recapitulated age at onset distributions typical of HD.

Conclusions: Stochastic cell death is unlikely to explain an appreciable fraction of variability in age at onset of disease. Alternatively, variability in age at onset of disease can be readily explained by variability in factors such as rate of neuronal loss and symptomatic susceptibility to the extent of that cell loss. Age at onset given polyglutamine repeat length is not determined by an uninformative stochastic process, but rather is a valuable phenotypic endpoint for investigations of environmental and background genetic factors affecting the expression of HD.

\section{POSTER 22}

Automated, whole-brain MRI analysis detects premanifest HD neurodegeneration within a period of 1 year.

A. Majid, ${ }^{1}$ D. Stoffers, ${ }^{2}$ S. Sheldon, ${ }^{1}$ S. Hamza, ${ }^{1}$ J. Goldstein, ${ }^{1}$ J. Corey-Bloom, ${ }^{1}$ and A. Aron. ${ }^{1}{ }^{1}$ University of California, San Diego (UCSD), La Jolla, CA, USA and ${ }^{2}$ Netherlands Institute for Neurosciences, Amsterdam, Netherlands.

Objective: Intense efforts are underway to evaluate neuroimaging measures as potential biomarkers for neurodegeneration in gene-positive premanifest Huntington's disease (preHD). Such biomarkers are crucial for future studies of neuroprotection. Here, we used a completely automated, longitudinal analysis method to compare structural magnetic resonance imaging (MRI) scans in preHD and controls.

Methods: Using a longitudinal design with a 1-year interval, we acquired $\mathrm{T}_{1}$-weighted MRI scans in 35 preHD individuals and 22 age-matched controls. We used the SIENA software tool (Structural Image Evaluation, using Normalization, of Atrophy) to yield both overall Percentage Brain Volume Change (PBVC) and a map of voxel-level changes in atrophy.

Results: We found significantly greater atrophy in preHD compared to controls over 1 year (Mean PBVC controls= $-0.149 \%$; preHD $=-0.388 \%$; $p=0.031$, Cohen's $d=0.617$ ). For a preHD subgroup that was estimated to be close to disease onset, atrophy was over three times greater than controls (Mean PBVC close-to-onset preHD $=-0.510 \% ; p=0.019$, Cohen's $d=$ 0.920 ). This atrophy was evident at the voxel level in periventricular regions-consistent with the well-established basal ganglia atrophy in preHD.

Interpretations: Automatic whole-brain analysis of structural MRI can reliably detect disease progression in preHD over a 1year period. These results were attained with a readily available imaging analysis tool-SIENA-which is observer independent, automatic, and robust with respect to image quality, slice thickness, and different pulse sequences. The MRI biomarker approach develop here could be readily applied to evaluate whether neuroprotection is effective in preHD.

\section{POSTER 23}

Traditional and Novel Recruitment Strategies Utilized in the Functional Rating Scale Task Force for pre-HD (FuRST-pHD) Project.

K. Evans, ${ }^{1}$ L. Goodman, ${ }^{2}$ J. Giuliano, ${ }^{3}$ M. Guttman, ${ }^{4}$ W. Meschino, ${ }^{5}$ J. Paulsen, ${ }^{6}$ H. Rickards, ${ }^{7}$ D. van Kammen, ${ }^{3}$ O. Yastrubetskaya, ${ }^{8}$ and A. Vaccarino. ${ }^{1}{ }^{1}$ Ontario Cancer Biomarker Network, Toronto, Canada, ${ }^{2}$ Huntington's Disease Drug Works, Lake Forest Park, WA, USA, ${ }^{3}$ CHDI Foundation, Princeton, NJ, USA, ${ }^{4}$ Center for Movement Disorders, Toronto, Canada, ${ }^{5}$ North York General Hospital, Toronto, Canada, ${ }^{6}$ University of Iowa, Iowa City, IA, USA, ${ }^{7}$ Queen Elizabeth Psychiatric Hospital, Birmingham, UK and ${ }^{8}$ St. Vincents Hospital, Melbourne, Australia.

Objective: To describe traditional and novel recruitment strategies utilized in the Functional Rating Scale Task Force for pre-HD (FuRST-pHD) Project.

Background: FuRST-pHD is a CHDI-sponsored multinational, multidisciplinary initiative with the overall goal of developing a sensitive, reliable, and valid rating scale to measure symptoms and their functional impact in patients at the pre$\mathrm{HD}$ and early stages of the disease. This project is based on a series of structured and sequenced interviews that identify those question areas (items) that have greatest functional impact in this unique patient population.

Methods: Testing of first items began in March of 2009 with the initial intent of using large ongoing longitudinal observation studies including PREDICT HD, REGISTRY, and TRACK-HD for patient recruitment. To complement recruitment within these ongoing studies, novel recruitment strategies have been utilized. Rather than inviting all sites, five sites with history of more rapid recruiting were chosen as Accelerated Recruitment Centers for FuRST-pHD testing. This led to recruitment of additional subjects separate from those other ongoing studies. Subsequently, a third method that utilizes regional family advocate grass-roots recruiting was initiated.

Results: Novel recruitment strategies employed within FuRSTpreHD have been successful for both increasing recruitment and accelerating item testing.

Conclusions: Historically the vast majority of participants for HD clinical studies and trials have been drawn from HDSA Center of Excellence (COE) and Huntington Study Group sites. Although recruitment rate has been slow there has previously been the ability to complete the majority of trials. However as multiple concurrent trials are now offered and compete for a limited number of COE participants within this patient population, it is increasingly vital to recruit outside of the standard COE model. Novel recruitments methods utilized in FuRST-pHD can serve as successful models for more rapid and extensive recruitment of targeted populations. 
POSTER 24

Usefulness of cognitive and psychiatric measures in genepositive pre-manifest Huntington's disease.

S. Gluhm, J. Goldstein, S. Edland, M. Generoso, A. Majid, A. Aron, and J. Corey-Bloom. University of California, San Diego, La Jolla, CA, USA.

Objective: To examine the usefulness of various cognitive and psychiatric measures to assess change in subjects with genepositive pre-manifest Huntington's disease (preHD).

Background: The Unified Huntington's Disease Rating Scale (UHDRS) is a standardized clinical measure commonly used to assess cognitive and behavioral functioning in HD. The UHDRS has also been used to examine functioning in preHD; however, less is known about its sensitivity and change over time in this population. Some degree of impulsivity is quite common in HD, but few studies have specifically examined this or other psychiatric manifestations in preHD.

Methods: Thirty-nine preHD and 23 normal control (NC) subjects were administered the UHDRS cognitive battery, additional neuropsychological tests, the Barratt Impulsivity Scale (BIS), and the Symptom Checklist-90-Revised (SCL-90$\mathrm{R})$ Inventory at baseline and approximately 1 year later.

Results: preHD and $\mathrm{NC}$ subjects were well matched with regard to age (preHD $=40.5$ years, $\mathrm{NC}=39.1$ years) and education (preHD $=15.6$ years, $\mathrm{NC}=15.8$ years) at initial visit. Compared to $\mathrm{NC}$, preHD subjects declined on the MMSE over 1 year $(p=$ $0.057)$. In addition, they scored significantly lower on the Symbol Digit Modalities test across timepoints $(p=0.015)$. Compared to $\mathrm{NC}$, preHD subjects demonstrated significantly higher impulsivity on the attention subscale of the BIS ( $p=$ $0.039)$. They scored significantly higher than NC subjects on SCL-90-R global indices, and all primary symptom dimensions (depression, obsessive-compulsive, interpersonal sensitivity, anxiety, hostility, phobic anxiety, paranoid ideation, psychoticism), except somatization, across the two time points (all $p<0.05$ ).

Conclusion: The MMSE, Symbol Digit Modalities test, BIS, and SCL-90-R may be useful measures for distinguishing early symptoms and tracking change over time in preHD. Further studies will be needed to confirm and extend these findings.
Study supported by: UCSD Huntington's Disease Society of America Center of Excellence; UCSD Shiley-Marcos Alzheimer's Disease Research Center NIH P50 AG 005131 and NIH RO3 AG 034439 (S.D.E.)

\section{POSTER 25}

Rate of striatal atrophy increases with CAG repeat length. E. Aylward, ${ }^{1}$ D. Liu, ${ }^{2}$ P. Nopoulos, ${ }^{2}$ C.Ross, ${ }^{3}$ R. Pierson, ${ }^{2}$ J. Mills, ${ }^{2}$ J. Paulsen, ${ }^{2}$ and the PREDICT-HD Investigators. ${ }^{1}$ Seattle Children's Research Institute, Seattle, WA, USA, ${ }^{2}$ University of Iowa, Iowa City, Iowa, USA, and ${ }^{3} J o h n s$ Hopkins University, Baltimore, MD, USA.

Although evidence was inconclusive until recently, it is now fairly clear that clinical progression is faster in individuals with higher CAG repeat lengths (Rosenblatt et al., 2006). Evidence for faster progression of striatal atrophy with higher CAG repeat length is not as clear. We conducted a study examining the association between age and striatal volume in each of eight groups defined by CAG repeat length $(\leq 39,40,41,42,43,44$ or 45,46 , and $\geq 46)$. The study included baseline MRI data from 475 participants of the PREDICT-HD study (CAG repeat length range $=38-61$ ), all of whom were prodromal at the time of the baseline scan. Each CAG group had at least 22 subjects. The slope defining the linear regression between striatal volume (corrected for intracranial volume) and age for each group was entered into a regression with $\mathrm{CAG}$ group. The slope for each group was highly associated with CAG group $(r=0.995 ; p<0.0001)$. Previous analysis of longitudinal data from the PREDICT study (Aylward et al., submitted) also revealed a significant association between $\mathrm{CAG}$ repeat length and rate of change for caudate $(t=-2.64, p=0.009)$ and total striatum $(t=-2.32, p=0.02)$; there was a trend toward a significant association for putamen $(t=-1.80, p=0.07)$. No significant associations were observed, however, for any other regions, with $p$ values ranging from 0.24 to 0.85 . Taken together, these findings yield convincing evidence that rate of striatal atrophy is significantly faster in individuals with higher CAG repeat lengths. 


\section{LATE-BREAKING RESEARCH}

\section{POSTER 26}

\section{The histone demethylase SMCX/JARID1C as a therapeutic} target for Huntington's Disease.

M. Vashishtha, A. Lau, I. Kratter, I. Martins, B. Davidson, S. Finkbeiner, and L. M. Thompson. University of California, Irvine, CA, USA.

Transcriptional down-regulation of cortical and striatal genes such as BDNF, preproenkephalin and dopamine receptor is an early marker of huntington's (HD) pathology. We find that an epigenetic mark of active transcription, trimethylation of lysine four residue of histone $\mathrm{H} 3$ ( $\mathrm{H} 3 \mathrm{~K} 4 \mathrm{me} 3)$, is decreased at the BDNF and Penk1 promoters in cortices of 12 week old R6/2 mice. We also find that the demethylase specific for $\mathrm{H} 3 \mathrm{~K} 4 \mathrm{me} 3$, SMCX/JARID1C, is increased in cortex of R6/2 mouse model of HD. Hence, we hypothesize that increased levels of SMCX/ JARID1C and increased binding at promoters due to interaction with repressors such as REST are a critical event in HD pathology. We tested the role of SMCX/JARID1C in transcriptional dysregulation by ShRNA mediated knockdown of the demethylase in primary cortical neurons. Down-regulation of SMCX/JARID1C in both wild type as well. BACHD primary cortical neurons increase transcription of BDNF, DRD2, and Penk1. Transduction of primary cortical neurons with viruses expressing expanded polyglutamines resulted in downregulation of several neuronal genes and co-transduction with lentiviruses expressing ShRNA to SMCX/JARID1C led to a reversal of the transcriptional down-regulation. Preliminary results also show that silencing of SMCX/JARID1C rescues cytotoxic effects of expanded polyglutamines in primary cortical neurons using single cell microscopic assay.

Thus down-regulation of SMCX/JARID1C may provide a novel therapeutic target in treatment of HD.

We are currently testing the efficacy of knocking down SMCX/JARID1C in reversing the phenotype in BACHD mouse model of HD.

\section{POSTER 27}

The Importance of Nursing Assessment in the Patient with Huntington's Disease.

\section{E. Robinson. York College of Pennsylvania, York, PA, USA.}

The onset of symptoms in a person with Huntington's Disease (HD) may be insidious. It is imperative that the nurse performs a thorough overall assessment of the patient to identify even the most subtle deviations from the norm. The neurological and mental health assessments are especially vital in identifying the signs and symptoms of HD. In the early stages of the disease, initial symptoms may include loss of balance and coordination, such as dropping items and tripping over one's feet, and mood swings, irritability, and depression. Specific specialized assessments of the neurological system such as the Romberg test may adequately assess balance deficits. Examination of coordination can be done with a rapid alternating movement test. A person with HD is seven times more likely to commit suicide than his peers due to depression. A thorough mental health assessment should include the Beck Depression Inventory, as well as questions regarding interpersonal relationships, stressors, anger, alcohol and drug abuse, and anxiety. Utilizing prudent nursing skills to identify these early signs and symptoms, and teaching your patient interventions to assist with the management and alleviation of the symptoms may greatly help patients and caretakers cope with the onset of this disease.

\section{POSTER 28}

Intermediate CAG repeats: Analysis of the Cooperative Huntington's Observational Research Trial.

A. Ha, A. Davidson, and J. Jankovic. Baylor College of Medicine, Parkinson's Disease Center and Movement Disorders Clinic, Houston, TX, USA.

Background: In recent years, there has been emerging evidence to suggest the presence of clinical and neuro-pathological manifestations of Huntington's disease (HD) in individuals with intermediate length $\mathrm{CAG}$ repeats. In addition to welldocumented cases of HD phenotype in patients with intermediate CAG repeat, the Prospective Huntington Disease At-Risk Observational Study (PHAROS) found that patients with intermediate $\mathrm{CAG}$ lengths overlapped with those diagnosed as HD ( $\geq 37$ CAG repeats) on the Unified Huntington's Disease Rating Scale (UHDRS) behavioral measures.

Objectives: We aimed to further define the clinical characteristics of subjects who possess CAG repeat lengths in the intermediate range.

Methods: Data from the Cooperative Huntington's Observational Research Trial (COHORT) was analyzed. Subjects were categorized into normal, intermediate (27-36 CAG repeats) and HD groups, consistent with guidelines used in the PHAROS study. The motor, cognitive, and behavioral UHDRS scores were then compared between the intermediate and normal groups. Analyses were additionally performed on individuals in the intermediate group who had CAG repeat lengths in the upper range (31-36 inclusive), and these were also compared with the normal group.

Results: There were statistically significant differences in UHDRS scores at baseline between normal and intermediate length CAG repeats. These differences were most prevalent in the motor component of the UHDRS. Analysis of a subgroup of individuals in the upper range of intermediate $\mathrm{CAG}$ repeats revealed additional differences compared to the normal $\mathrm{CAG}$ group.

Conclusion: Our findings of motor and behavioral abnormalities in individuals with intermediate $\mathrm{CAG}$ repeats indicate that current guidelines for genetic counseling and diagnosis of $\mathrm{HD}$ should be re-examined.

\section{POSTER 29}

CSF peptide profiling in Huntington's disease for the identification of peptide candidates.

S. Süßmuth, ${ }^{1}$ H.-D. Zucht ${ }^{2}$ P. Budde, ${ }^{2}$ H. Selle, ${ }^{2}$ D. Ecker, ${ }^{1}$ and G.B. Landwehrmeyer. ${ }^{1}{ }^{1}$ Department of Neurology, University of Ulm, Germany and ${ }^{2}$ Proteome Sciences $R \& D$ (formerly Digilab BioVisioN), Frankfurt am Main, Germany.

Objective: Cerebrospinal fluid (CSF) is a promising source of biochemical markers in neurodegenerative diseases. The aim of 
the study was to identify new biomarker candidates by comparison of the CSF peptide profile between patients with Huntington's disease (HD) and normal controls.

Methods: Using peptide profiling by means of RP-HPLC and MALDI-TOF mass spectrometry followed by product ion database searches, we compared CSF samples from patients with different stages of HD (early stage, $n=13$; mid-stage, $n=$ $15)$ with those from normal controls $(n=16)$. HD patients were diagnosed in the university hospital of Ulm, Germany, and the severity of the disease was rated by using the UHDRS total functional capacity (TFC).

Results: After removal of redundant entries, a total of 121 putative biomarker candidates were found to be significantly differentially regulated. The targeted analysis of the statistical list of interest candidates resulted in the successful identification of 24 distinct peptides, which derived from 12 different precursor proteins. Eight peptides showed a correlation to the TFC score. Interestingly, two precursor proteins of the identified peptides (Proenkephalin and Cholecystokinin) have already been linked by histopathological findings to the phathophysiology of HD before.

Conclusions: Peptidomics analysis of CSF samples was successfully performed to discover novel biomarker cadidates for HD. Because some peptides showed a correlation to the severity of the disease, these might be suitable for disease staging. However, it is mandatory to consider the function of the blood-CSF barrier by determination of the CSF/serum ratio of albumin especially for the interpretation of biomarkers that are up-regulated in CSF. Although we present preliminary data, differentially expressed precursor proteins that have been previously described in the literature in the context of HD would support the validity of our results. Next steps include validation of selected candidates by immunoassays and verification of the results in an independent sample set.

\section{POSTER 30}

Tetrabenazine-Associated Adverse Events Compared During Initial Dosage Adjustment and Maintenance Phases of the Tetra-HD Trial.

F. Marshall, ${ }^{1}$ R. Dorsey, ${ }^{1}$ I. Shoulson, ${ }^{1}$ and K. ClarenceSmith. ${ }^{2}{ }^{1}$ University of Rochester Medical Center, Rochester, NY, USA and ${ }^{2}$ KM Pharmaceutical Consulting LLC, Washington, DC, USA.

Tetrabenazine (TBZ) is the only FDA-approved treatment for chorea associated with Huntington disease (HD). Optimal dosing varies by patient, ranging from 12.5 to $100 \mathrm{mg} /$ day, and is determined by a slow upward adjustment followed as needed by downward adjustment. Employing a post-hoc analysis, we sought to determine when adverse events (AEs) would most commonly occur during the 12-week, doubleblind, parallel-group, placebo-controlled study TETRA-HD. ${ }^{1}$ TBZ dosage was increased weekly in 12.5 -mg increments (corresponding to one tablet) during the first 7 weeks of the study (adjustment period), starting at $12.5 \mathrm{mg}$ /day (up to a maximum dosage of $100 \mathrm{mg} /$ day), until desired efficacy was achieved or dosage-limiting AEs supervened. Investigators were instructed to down-titrate TBZ to a dosage at which AEs resolved or were tolerable (in the event of dosage-limiting AEs). This was followed by a 5 -week maintenance period. In the TBZ group, $91 \%$ of HD patients experienced one or more AEs after 9 weeks ( 7 weeks of initial dosage adjustment plus 2 weeks to allow for onset of AEs), but only $35 \%$ of patients continued to experience AEs 3 weeks later during the maintenance phase of the study. A total of 28 of 54 participants randomized to TBZ discontinued upward dosage adjustment because of an AE. Of these, 24 had achieved an anti-chorea effect with TBZ. Decreasing dosage to resolution of AEs for these 24 participants resulted in sustained efficacy for 14, reduced efficacy for six, and loss of efficacy for four. AEs associated with TBZ, therefore, appear to be primarily associated with upward TBZ dosage adjustment. Downward dosage adjustment resulted in reduced AEs and maintained anti-chorea efficacy for most patients.

${ }^{1}$ Marshall $\mathrm{F}$ and the Huntington Study Group. Neurology. 2006;66: 366-72.

\section{POSTER 31}

\section{Preventive Cancer Screening Among Individuals in CO- HORT.}

E. Oster ${ }^{1}$ and E. R. Dorsey, ${ }^{2}$ on behalf of Huntington Study Group COHORT Investigators. IUniversity of Chicago, Chicago, IL, USA and ${ }^{2}$ University of Rochester, Rochester, NY, USA.

Individuals at risk for or with Huntington disease (HD) face a limited life expectancy relative to individuals in the general population. These individuals are therefore less likely to experience mortality from non-HD causes. This potentially shortened life expectancy may impact willingness to invest in non-HD preventive health behaviors such as cancer screening. We used data from the COHORT population to evaluate whether individuals at risk for HD or known to carry the huntingtin mutation were less likely to engage in cancer screening than the general population. We initially focused on mammography. Individuals in COHORT were asked in the Analysis of Life Decision Substudy whether they had ever had a mammogram. For comparison, we used individuals from the Behavioral Risk Factor Surveillance Survey (BRFSS), which asked the same question. We used data on 31 asymptomatic individuals in COHORT who are known to carry the huntingtin mutation and 65 asymptomatic individuals who are at risk for HD but have not pursued genetic testing. We compared these individuals to 253,897 individuals from the BRFSS who are not at risk for HD. The proportion of individuals who reported ever having a mammogram was $48.3 \%$ among the individuals who know they carry the huntingtin mutation, $60.0 \%$ among those at risk and $79.0 \%$ in the BRFSS sample. After adjusting for differences in age, gender and education, individuals who carry the huntingtin mutation are $11.8 \%$ less likely to have had a mammogram than those in the general population $(p=0.026)$ and at-risk individuals are $7.3 \%$ less likely than those in the general population $(p=0.05)$. There are no significant differences in screening behavior between those at risk and those who carry the huntingtin mutation. Individuals who are at risk for HD or carry the huntingtin mutation appear to be less likely than the general population to invest in mammography.

**Dorsey acknowledges Funding from the Robert Wood Johnson Physician Faculty Scholars Program

\section{POSTER 32}

A Randomized, Double-Blind Placebo-Controlled Trial of ACR16 in Huntington's Disease.

HART Investigators of the HSG (A. McGarry: corresponding author; K. Kieburtz: presenting author). University of Rochester, Rochester, NY, USA.

Please see Platform Presentation above for abstract body. 
POSTER 33

Preliminary Longitudinal Modeling of UHDRS Total Motor Scores from the COHORT Database.

J. Warner. CHDI Foundation, Princeton, NJ, USA.

We report the results of preliminary mixed effects modeling of the UHDRS Total Motor Score (TMS) from the 2010 cut of the COHORT database. The analysis included 1,502 observations on 499 subjects ( 170 gene negative controls, 71 gene positive pre-HD gene positive subjects and 258 patients who were diagnosed with $\mathrm{HD}$ at baseline). In this sample, TMS was distributed with mean= $24, \mathrm{SD}=25, \min =0$, and $\max =121$. All patients had three or more visits recorded in the database. The dependant variable was logit $((\mathrm{TMS}+1) / \mathrm{M})$. Where $M$ is an upper bound on the TMS score (taken to be 130). Predictors were age (for all patients) and genetic burden (for gene positive patients). Two final models were considered, one model that included an indicator for diagnostic status (one for patients diagnosed with $\mathrm{HD}$, zero otherwise) and one that did not. A random effect for subject was included in both models. No statistically significant random effect for age or genetic burden could be found. Genetic burden was defined as age * (cag length- $L$ ) where $L$ was estimated from the data. The estimated values for $L$ were only slightly lower than the standard values of 35.5. All fixed effects were significant at the 0.0001 level. Combined fixed and random effects explained $89 \%$ of the variance in the dependent variable in both models. Implications for the design of clinical trials for disease modifying compounds and the search for genetic modifiers of HD are discussed. The model fit was hampered by the inherent non-normality of the TMS (which takes on only integer values and included 359 zeros and 102 ones in the current sample). More refined modeling is being planned that will be more consistent with these features of the data.

\section{POSTER 34}

Engaging patients with Huntington's Disease in expression through fine art 2007-2010.

Linda Bounds. Edgemoor DPSNF, Santee, CA, USA.

Background/Objective: Edgemoor is a county-run distinct part skilled nursing facility that, in addition to caring for patients with wide ranging physical and psychiatric problems, has become a referral site for patients with Huntington's disease. Patients with middle to advanced Huntington's disease often are not readily engaged in activities due to severe chorea, short attention span and neurobehavioral challenges.

Process: In October 2007, Edgemoor invested in a customized group-painting program. This "healing heARTs" program was designed to assist patients to express their feelings about their lives, to reduce behavioral problems, and to assist with the emotional transition of the upcoming move into a new building.

Results/Analysis: The "healing heARTs" program has had visible results with particular benefits to patients with Huntington's disease. These patients, who were often not wellintegrated into the community, have been encouraged and honored for their contributions to group painting efforts despite their severe physical limitations. Individual sessions with patients with Huntington's disease have resulted in art that is both of high quality, and emotionally satisfying for the patients and their loved ones. Individual and group art projects by patients with advanced Huntington's disease are displayed with stories about the artists and their experience.
Conclusions: Patient involvement in a "specialized" art program assisted in the development of a therapeutic milieu, reducing behavioral problems and enhancing quality of life.

Authors/Affiliations: Linda Bounds, Artist and Educator

With Special Thanks to: Rebecca Ferrini, MD, MPH, CMD, Medical Director, Edgemoor, Gwenmarie Hilleary, FACHE Administrator, Edgemoor Distinct Part SNF, County of San Diego, Santee, California, Pam Smith, Aging and Independence Services Director, Gene Hand, Volunteer, and Participants Anthony, Britia, Jessica, Ramona, and Tracy.

\section{POSTER 35}

Project AWARE*: What are the Barriers to Patient Participation in Clinical Trials in Huntington Disease? A View from the Sites.

K. Anderson, ${ }^{1}$ J. Megens, ${ }^{2}$ J. Giuliano, ${ }^{3}$ J. Goldstein, ${ }^{4}$ B. Hennig, ${ }^{5}$ E. Kayson, ${ }^{6}$ S. Kinel, ${ }^{6}$ L. McCarthy, ${ }^{6}$ P. Auinger, ${ }^{6}$ and of the Project AWARE Ability Team. ${ }^{1}$ University of Maryland, Baltimore, MD, USA, ${ }^{2}$ London Health Sciences Centre, London, ON, Canada ${ }^{3}$ CHDI Foundation, Princeton, NJ, USA, ${ }^{4}$ University of San Diego, San Diego, CA, USA, ${ }^{5}$ University of Connecticut Health Center, Farmington, CT, USA, and ${ }^{6}$ University of Rochester, Rochester, NY, USA.

Objective: To study site-identified barriers to clinical trial participation by Huntington disease (HD) patients and their families. Results will guide development of future clinical trials to foster more participation.

Background: It has been problematic to recruit adequate numbers of HD patients for recent clinical trials. This is despite the fact that there is no known cure for HD and advances in the understanding of HD neuropathology have brought new agents to clinical trials.

Methods: All Huntington Study Group (HSG) research sites were sent an electronic survey with 8 multiple part questions. Two questions addressed respondent demographics, and 6 queried barriers to subject participation in clinical trials at HSG sites. Responses included rankings of: barriers, tools that sites use for recruitment, and potential site resources.

Results: $73 / 83$ sites responded to the survey at the time of data analysis. Coordinators (66\%) and Investigators (34\%) completed surveys. The most commonly cited barriers to participation, ranked as "most problematic", were: 1) exclusionary medications, 2) travel time/distance, 3) participant not having caregiver. The most used recruitment tools were direct talks with potential subjects at clinic (88\%) and support group and other talks to increase awareness (80\%). Coordinators ranked "More staffing at the site" as the most useful site resource. Investigators felt that both "More time at the site to dedicate to recruitment" and "More staffing at the site" were equally useful resources.

Conclusions: Commonly cited barriers to subject participation were identified as exclusionary medications, travel time and caregiver availability. Sites identified the need for more time and staffing to recruit for HD clinical trials. If progress is to be made in HD drug development, these issues will need to be addressed directly by sponsors in the design of future studies, by institution staffing requirements and more broadly at a policy level.

*Project AWARE to improve Awareness, Willingness and Ability for Research and Enrollment 
POSTER 36

What can a skilled physical therapist offer in management of mid and late stage Huntington's Disease?

I. Busacco, ${ }^{1}$ R. Ferrini, ${ }^{1}$ and M. E. Dellefield. ${ }^{2}{ }^{1}$ Edgemoor Hospital DPSNF, Santee, CA, USA and ${ }^{2}$ VA San Diego Medical Center, San Diego, CA, USA.

Huntington's disease is a rare, progressive neurological disorder, often lasting more than two decades, characterized by abnormal movements and neuro-cogntiive decline and passed through an autosomal dominant transmission. Patients in the mid-to late stages of the illness manifest severe gait disturbances, heightened fall risk and challenges in seating and maintaining safety-many require institutionalization. Our facility cared for more than 50 residents with HD from 2000 to 2008 and currently has 24 inpatients with the disease. Having a larger cohort of patients has allowed us to make some generalizations about care needs and practices. In general, individuals with HD have difficulty benefiting from skilled physical therapy because of the progressive nature of the disease, the difficulty in attention and cooperation, and the nature of the gait disturbances. However, skilled therapists are an invaluable consultant to the care team in problem solvingparticularly around maintaining safety. We write here about our experiences with these patients and provide suggestions as to how a skilled physical therapist can improve the care and function of patients with this devastating disease. 